Pacific Journal of Mathematics

ROOTS OF THE EULER POLYNOMIALS 


\title{
ROOTS OF THE EULER POLYNOMIALS
}

\author{
F. T. HOWARD
}

In this paper we prove some new theorems about the real and complex roots of the Euler polynomials. For each $n$ we show how the real roots of $E_{n}(x)$ are distributed in the closed interval $[1,3]$. We also show how the real roots of $E_{n}(x)$ are distributed in the arbitrary interval $[m, m+1]$ for $n$ sufficiently large. Finally, we prove that if $a$ and $b$ are nonzero rational numbers and $c$ is a square-free integer, then $E_{n}(x)$ has no roots of the form $a \sqrt{ } \bar{c}, c \neq 1$, or $a+b \sqrt{ } \bar{c}, c$ even, or $a+b i, a$ and $b$ integers.

1. Introduction. The Euler polynomial $E_{n}(x)$ degree $n$ can be defined as the unique polynomial satisfying

$$
E_{n}(x+1)+E_{n}(x)=2 x^{n} \quad(n \geqq 0) .
$$

These polynomials have been extensively studied; see [3, Chapter VI] and [4, Chapter II] for example. The first fifteen Euler polynomials are listed in [5, p. 477].

In this paper we are primarily concerned with the real roots of $E_{n}(x)$, though we also prove a few results about the complex roots. It is well known that if $n$ is even, $n>0$, then the only real roots of $E_{n}(x)$ in the closed interval $[0,1]$ are 0 and 1 , while if $n$ is odd the only real root in $[0,1]$ is $1 / 2$. Brillhart [1] has pointed out that these are the only complex roots in the "critical strip" of all complex numbers $x+i y, 0 \leqq x \leqq 1$. In the same paper Brillhart proved that $E_{5}(x)$ is the only Euler polynomial with a multiple root and that the Euler polynomials have no rational roots other than $0,1,1 / 2$.

The main results in this paper are:

(1) On the closed interval $[1,3]$ we show how the real roots of $E_{n}(x)$ are distributed for each $n$.

(2) On each interval $[m, m+1], m>0$, we show how the real roots of $E_{n}(x)$ are distributed for $n$ sufficiently large.

(3) Let $a$ and $b$ be nonzero rational numbers and let $c$ and $d$ be square-free integers. The polynomial $E_{n}(x)$ has no roots of the form $a \sqrt{c},(c \neq 1), a+b \sqrt{c}$ (c even), $a \sqrt{d}+b \sqrt{c} i$ ( $c$ and $d$ of different parity); or $a+b i$ ( $a, b$ integers).

It is pointed out that results similar to (3) are also true for the Bernoulli polynomials. 
2. Preliminaries. Throughout this paper we use the notation of Nörlund [4]. The following are well-known identities:

$$
\begin{aligned}
& E_{n}^{\prime}(x)=n E_{n-1}(x) \quad(n>0), \\
& E_{n}(1-x)=(-1)^{n} E_{n}(x), \\
& E_{n}(x)=\sum_{s=0}^{n}\left(\begin{array}{l}
n \\
s
\end{array}\right) 2^{-s} C_{s} x^{n-s}
\end{aligned}
$$

where

$$
C_{s-1}=\frac{2^{s}\left(1-2^{s}\right)}{s} B_{s}
$$

In formula (2.3), $B_{s}$ is the $s^{\prime}$ th Bernoulli number (see [4, pp. 17-23]). If $s$ is odd, $s>1$, then $B_{s}=0$. If $s$ is even, $s>0$, then the denominator of $B_{s}$ is even and square-free.

The Euler polynomials are related to, and often studied in conjunction with, the Bernoulli polynomials $B_{n}(x)$ [3, Chapter V], [4, Chapter II]. The Euler and Bernoulli polynomials are related by

$$
n E_{n-1}(x)=2^{n}\left[B_{n}\left(\frac{x+1}{2}\right)-B_{n}\left(\frac{x}{2}\right)\right]
$$

The numbers $E_{2 k}$ defined by

$$
E_{2 k}=2^{2 k} E_{2 k}(1 / 2)
$$

are known as the Euler numbers and have the following properties:

$$
\begin{gathered}
(-1)^{k} E_{2 k}>0 \\
(-1)^{k}(2 \pi)^{2 k+1} E_{2 k}=2^{4 k+3}(2 k) ! \sum_{n=0}^{\infty}(-1)^{n}(2 n+1)^{-2 k-1} .
\end{gathered}
$$

The first sixty Euler numbers, as well as the first sixty Bernoulli numbers and the first fifteen Bernoulli polynomials are listed in [5, pp. 477-479].

From (2.7) and inequalities proved in [3, pp. 294-295, 302], it follows that for $k>0$

$$
(2 k-1) ! / 4^{2 k-1}<\left|E_{2 k-1}(0)\right|<2(2 k-1) ! / 3^{2 k-1},
$$

$$
(2 k) ! / 2^{2 k}<\left|E_{2 k}\right| \text {, }
$$$$
(2 \pi)^{2}\left|E_{2 k}\right|>16(2 k)(2 k-1)\left|E_{2 k-2}\right| \text {. }
$$ 
Finally, we shall use the following formulas which are derived by expanding $E_{n}(x)$ into a series about $x=a$ and then using (2.3).

$$
\begin{aligned}
& E_{2 k}(a+b \sqrt{d}) /(2 k) ! \\
& =\sum_{r=0}^{k} \sum_{s=0}^{2 r} d^{k-r} b^{2 k-2 r} a^{2 r-s} C_{s} / 2^{s}(2 k-2 r) ! s !(2 r-s) ! \\
& +\sqrt{d} \sum_{r=0}^{k-1} \sum_{s=0}^{2 r+1} d^{k-r-1} b^{2 k-2 r-1} a^{2 r+1-s} C_{s} / 2^{s}(2 k-2 r-1) ! s !(2 r+1-s) !
\end{aligned}
$$

(2.12) $E_{2 k+1}(a+b \sqrt{d}) /(2 k+1)$ !

$$
\begin{aligned}
= & \sum_{r=0}^{k} \sum_{s=0}^{2 r+1} d^{k-r} b^{2 k-2 r} a^{2 r+1-s} C_{s} / 2^{s}(2 k-2 r) ! s !(2 r+1-s) ! \\
& +\sqrt{d} \sum_{r=0}^{k} \sum_{s=0}^{2 r} d^{k-r} b^{2 k+1-2 r} a^{2 r-s} C_{s} / 2^{s}(2 k+1-2 r) ! s !(2 r-s) !
\end{aligned}
$$

The numbers $C_{s}$ in (2.11) and (2.12) are defined by (2.3).

3. Distribution of the real roots of $E_{n}(x)$. Inkeri [2] has shown how the positive real roots of the Bernoulli polynomials are distributed outside of the interval $[0,1]$. To the author's knowledge this has not been attempted for the Euler polynomials. By (2.2), if we restrict our attention to the positive real roots we can determine how all the roots are distributed. Thus we shall only consider the positive real roots and we shall use (1.1), which tells us that if $E_{n}(a)<0$ then $E_{n}(1+a)>0$.

First we note that if $m$ is a positive integer we have, by (1.1),

$$
\begin{aligned}
& \text { (3.1) } E_{n}(m)=(-1)^{m} E_{n}(0)+2 \sum_{k=0}^{m-2}(-1)^{k}(m-1-k)^{n}, \\
& \text { (3.2) } E_{n}(m+1 / 2)=(-1)^{m} E_{n}(1 / 2)+2 \sum_{k=0}^{m-1}(-1)^{k}(m-k-1 / 2)^{n} .
\end{aligned}
$$

Since $E_{n}(0)=0$ if $n$ is even and $E_{n}(1 / 2)=0$ if $n$ is odd, we see that

$$
\begin{aligned}
& E_{n}(m)>0 \text { if } n \text { is even, } \\
& E_{n}(m+1 / 2)>0 \text { if } n \text { is odd. }
\end{aligned}
$$

Furthermore, by (2.3) and (3.1),

$$
E_{4 k+1}(m)>0 \text { if } m \text { is odd, }
$$




$$
E_{4 k+3}(m)>0 \text { if } m \text { is even. }
$$

By (2.6) and (3.2), we see that

$$
\begin{array}{ll}
E_{4 k+2}(m+1 / 2)>0 & \text { if } m \text { is odd, } \\
E_{4 k+4}(m+1 / 2)>0 & \text { if } m \text { is even. }
\end{array}
$$

THEOREM 3.1. Let $k>0$. Then $E_{4 k}(x)$ has exactly one real root $\alpha_{1}$ in the open interval $(1,2)$ and $3 / 2<\alpha_{1}<2$;

$E_{4 k+1}(x)$ has exactly one real root $\alpha_{2}$ in $(1,2)$ and $3 / 2<\alpha_{2}<2$;

$E_{4 k+2}(x)$ has no real roots in $(1,2)$;

$E_{4 k+3}(x)$ has exactly one real root $\alpha_{3}$ in $(1,2)$ and $1<\alpha_{3}<3 / 2$.

Proof. The proof for $E_{4 k}(x)$ is due to Brillhart [1]. By (3.1), (3.3) and (3.8), we know that $E_{4 k}(1)=0, E_{4 k}(2)=2, E_{4 k}(3 / 2)<0$. Furthermore, since $E_{4 k-2}(x)<0$ for $0<x<1$, we know $E_{4 k-2}(x)>0$ for $1<x<$ 2 . Thus, by (2.1), $E_{4 k}(x)$ is concave up for $1<x<2$ and has exactly one real root $\alpha_{1}$ in $(1,2), 3 / 2<\alpha_{1}<2$.

Now the theorem is true for $E_{5}(x)=(x-1 / 2)\left(x^{2}-x-1\right)^{2}$, so we examine $E_{4 k+1}(x)$ for $k \geqq 2$. We know that $E_{4 k+1}(1)>0, E_{4 k+1}(3 / 2)>0$ and $E_{4 k+1}(2)=2+E_{4 k+1}(0)$. Since by $(2.3) E_{4 k+1}(0)<0$ and since $E_{9}(0)=$ -15.5 , we see from $(2.8)$ that $E_{4 k+1}(2)<0$. We know there is exactly one number $\alpha_{1}$ in $(1,2)$ such that $E_{4 k+1}^{\prime}\left(\alpha_{1}\right)=0$. Hence $E_{4 k+1}(x)$ has exactly one real root $\alpha_{2}$ in $(1,2)$ and $\alpha_{2}>3 / 2$.

We know $E_{4 k+2}(x)>0$ for $1<x<2$ since $E_{4 k+2}(x)<0$ for $0<x<1$.

We know $E_{4 k+3}(1)<0, E_{4 k+3}(3 / 2)>0, E_{4 k+3}(2)>0$. Also $E_{4 k+3}^{\prime}(x)>0$ for $1<x<2$. Hence $E_{4 k+3}(x)$ has exactly one real root $\alpha_{3}$ in $(1,2)$ and $\alpha_{3}<3 / 2$.

It is clear from this proof that $\alpha_{3}<\alpha_{2}<\alpha_{1}$.

THEOREM 3.2. Let $k \geqq 4$. Then $E_{4 k+1}(x)$ has exactly one real root $\alpha_{4 k+1}$ in the closed interval [2,3] and $2<\alpha_{4 k+1}<5 / 2$;

$E_{4 k+2}(x)$ has exactly two real roots $\alpha_{4 k+2}^{(1)}, \alpha_{4 k+2}^{(2)}$ in [2,3] and $\alpha_{4 k+2}^{(1)}<$ $5 / 2<\alpha_{4 k+2}^{(2)}$

$E_{4 k+3}(x)$ has exactly one real root $\alpha_{4 k+3}$ in $[2,3]$ and $5 / 2<\alpha_{4 k+3}$;

$E_{4 k+4}(x)>0$ for $2 \leqq x \leqq 3$.

Furthermore, $\alpha_{4 k+2}^{(1)}<\alpha_{4 k+1}<5 / 2<\alpha_{4 k+3}<\alpha_{4 k+2}^{(2)}$.

Proof. We know $E_{4 k+1}(2)<0, \quad E_{4 k+1}(5 / 2)>0, \quad E_{4 k+1}(3)>0 . \quad$ By Theorem 3.1 we know that $E_{4 k}(x)<0$ for $1<x<\alpha_{1}$ and thus $E_{4 k+1}^{\prime}(x)>$ 0 for $2<x<1+\alpha_{1}$. Since $E_{4 k+1}(x)<0$ for $\alpha_{2}<x \leqq 2$ and since $\alpha_{2}<\alpha_{1}$, we see that $E_{4 k+1}(x)>0$ for $1+\alpha_{1}<x \leqq 3$. Thus $E_{4 k+1}(x)$ has exactly one real root $\alpha_{4 k+1}$ in [2,3] and $2<\alpha_{4 k+1}<5 / 2$.

We know that $E_{4 k+2}(2)>0, E_{4 k+2}(3)>0$ and we now show that for 
$k \geqq 3, E_{4 k+2}(5 / 2)<0$. We shall use (3.2) and (2.10). We first observe that - $E_{14}=199,360,981>2 \cdot 3^{14}$, so $E_{14}(5 / 2)<0$. Now by $(2.10)$ we see that if $\left|E_{2 t}\right|>2\left(3^{2 t}-1\right)$ then $\left|E_{2 t+2}\right|>2\left(3^{2 t+2}-1\right)$. Thus we have $E_{4 k+2}(5 / 2)<0$ for $k \geqq 3$. Now since $E_{4 k+2}^{\prime}(x)<0$ for $2<x<\alpha_{4 k+1}$ and $E_{4 k+2}^{\prime}(x)>0$ for $\alpha_{4 k+1}<x<3$, we see that $E_{4 k+2}(x)$ has exactly two real roots $\alpha_{4 k+2}^{(1)}, \alpha_{4 k+2}^{(2)}$ in $[2,3]$ and $\alpha_{4 k+2}^{(1)}<\alpha_{4 k+1}<5 / 2<\alpha_{4 k+2}^{(2)}$.

We know that $E_{4 k+3}(2)>0, E_{4 k+3}(5 / 2)>0$, and we now show that $E_{4 k+3}(3)<0$ for $k \geqq 4$. We shall use (3.1) and (2.8). We first note (by using tables) that $E_{19}(0)>2^{20}$, so by $(3.1) E_{19}(3)<0$. For $k=5$ we use (2.8) and we see that $\left|E_{23}(0)\right|>2^{24}$, and it is clear that for $k>5$ we have $\left|E_{4 k+3}(0)\right|>2^{4 k+4}$. Thus by (3.1) we see that $E_{4 k+3}(3)<0$ for $k \geqq 4$. We know that $E_{4 k+3}^{\prime}(x)>0$ for $2<x<\alpha_{4 k+2}^{(1)}$ and for $\alpha_{4 k+2}^{(2)}<x<3$, while $E_{4 k+3}^{\prime}(x)<0$ for $\alpha_{4 k+2}^{(1)}<x<\alpha_{4 k+2}^{(2)}$. It follows that $E_{4 k+3}(x)$ has exactly one real root $\alpha_{4 k+3}$ in [2,3] and $5 / 2<\alpha_{4 k+3}<\alpha_{4 k+2}^{(2)}$.

We know $E_{4 k+4}(2)>0, \quad E_{4 k+4}(5 / 2)>0, \quad E_{4 k+4}(3)>0$. Furthermore $E_{4 k+4}^{\prime}(x)>0$ for $2<x<\alpha_{4 k+3}$ and $E_{4 k+4}^{\prime}(x)<0$ for $\alpha_{4 k+3}<x<3$. It follows that $E_{4 k+4}(x)>0$ for $2 \leqq x \leqq 3$.

Since we assume $k \geqq 4$ in Theorem 3.2, we now look at the Euler polynomials $E_{n}(x)$ for $2 \leqq x \leqq 3$ and $n<17$. If $n \leqq 8, E_{n}(x)$ is a positive increasing function on $[2, \infty)$. With the aid of $(2.1),(3.1)-(3.8)$ and an electronic calculator, we have the following results for $9 \leqq n \leqq 16$ and the interval $[2,3]$ :

$E_{9}(x)$ has one real root $\alpha<5 / 2$ and is a positive, increasing function for $x>\alpha$.

$E_{10}(x)$ has two real roots $\alpha, \beta$ such that $\alpha<\beta<5 / 2$ and $E_{10}(x)$ is a positive increasing function for $x>\beta$.

$E_{11}(x)>0$ and is a positive, increasing function for $x>5 / 2$.

$E_{12}(x)$ is a positive, increasing function for $x \geqq 2$.

$E_{13}(x)$ has one real root $\alpha<5 / 2$ and is a positive, increasing function for $x>\alpha$.

$E_{14}(x)$ has two real roots $\alpha, \beta$ such that $\alpha<5 / 2<\beta$ and $E_{14}(x)$ is a positive increasing function for $x>\beta$.

$E_{15}(x)$ has two real roots $\alpha, \beta$ such that $5 / 2<\alpha<\beta$ and $E_{15}(x)$ is a positive, increasing function for $x>\beta$.

$E_{16}(x)>0$ and is a positive, increasing function for $x>3$.

In examining the real roots of $E_{n}(x)$ on a fixed positive interval $[m, m+1]$ we shall use the fact that if $n$ is sufficiently large, $E_{n}(0)$ and $E_{n}(1 / 2)$ dominate (3.1) and (3.2).

THEOREM 3.3. If $k>m^{2}$, then on the interval $[m, m+1]$ :

$E_{4 k+1}(x)$ has exactly one real root $\alpha_{4 k+1}\left(\alpha_{4 k+1}<m+1 / 2\right)$ if $m$ is even. $E_{4 k+1}(x)$ has exactly one real root $\beta_{4 k+1}\left(m+1 / 2<\beta_{4 k+1}\right)$ if $m$ is odd.

$E_{4 k+2}(x)$ has exactly two real roots $\alpha_{4 k+2}^{(1)}, \alpha_{4 k+2}^{(2)}\left(\alpha_{4 k+2}^{(1)}<m+1 / 2<\right.$ $\left.\alpha_{4 k+2}^{(2)}\right)$ if $m$ is even. $E_{4 k+2}(x)>0$ if $m$ is odd. 
$E_{4 k+3}(x)$ has exactly one real root $\alpha_{4 k+3}\left(m+1 / 2<\alpha_{4 k+3}\right)$ if $m$ is even. $E_{4 k+3}(x)$ has exactly one real root $\beta_{4 k+3}\left(m+1 / 2<\beta_{4 k+3}\right)$ if $m$ is odd.

$E_{4 k+4}(x)>0$ if $m$ is even. $E_{4 k+4}(x)$ has exactly two real roots $\beta_{4 k+4}^{(1)}$, $\beta_{4 k+4}^{(2)}\left(\beta_{4 k+4}^{(1)}<m+1 / 2<\beta_{4 k+4}^{(2)}\right)$ if $m$ is odd. Furthermore, $\alpha_{4 k+2}^{(1)}<\alpha_{4 k+1}<$ $\alpha_{4 k+3}<\alpha_{4 k+2}^{(2)}$ and $\beta_{4 k+4}^{(1)}<\beta_{4 k+3}<\beta_{4 k+1}<\beta_{4 k}^{(2)}$.

Proof. We have proved the theorem for $m=2$. Assume the theorem is true for any integer $t$ such that $2 \leqq t<m$.

Case 1. $m$ odd. We first examine the interval $[m-1, m]$; since $k>m^{2}$, it is clear that $k-1>(m-1)^{2}$. Thus, by our induction hypothesis, $E_{4 k-3}(x)$ has one real root $\alpha_{4 k-3}$ in $[m-1, m]$ and $\alpha_{4 k-3}<$ $m-1 / 2$. Also $E_{4 k-3}(m)>0$, so $E_{4 k-3}(m-1)<0$. Hence $E_{4 k-3}(x)>0$ for $m \leqq x \leqq 1+\alpha_{4 k-3}$. Also by our induction hypothesis, $E_{4 k-2}(x)$ has two real roots $\alpha_{4 k-2}^{(1)}, \boldsymbol{\alpha}_{4 k-2}^{(2)}$ in $[m-1, m]$ such that $\boldsymbol{\alpha}_{4 k-2}^{(1)}<\boldsymbol{\alpha}_{4 k-3}<m-1 / 2<$ $\alpha_{4 k-2}^{(2)}$, and since $E_{4 k-2}(m)>0, E_{4 k-2}(m-1)>0$, we have $E_{4 k-2}(x)>0$ for $1+\alpha_{4 k-2}^{(1)} \leqq x \leqq 1+\alpha_{4 k-2}^{(2)}$. Also by our induction hypothesis, $E_{4 k-1}(x)$ has one real root $\alpha_{4 k-1}$ in $[m-1, m]$ such that $m-1 / 2<\alpha_{4 k-1}<\alpha_{4 k-2}^{(2)}$. Also, $E_{4 k-1}(m-1)>0$, so $E_{4 k-1}(m)<0$. Thus $E_{4 k-1}(x)>0$ for $1+\alpha_{4 k-1} \leqq x \leqq$ $m+1$. Furthermore, $E_{4 k-1}(x)$ is concave up for $m \leqq x \leqq 1+\alpha_{4 k-3}$ and is increasing for $1+\alpha_{4 k-2}^{(1)} \leqq x \leqq 1+\alpha_{4 k-2}^{(2)}$ with $\alpha_{4 k-2}^{(1)}<\alpha_{4 k-3}<\alpha_{4 k-1}<\alpha_{4 k-2}^{(2)}$. Hence $E_{4 k-1}(x)$ has exactly one real root $\beta_{4 k-1}$ in $[m, m+1]$ and $\beta_{4 k-1}<$ $m+1 / 2$. Also, $E_{4 k-1}(x)<0$ for $m \leqq x<\beta_{4 k-1}, E_{4 k-1}(x)>0$ for $\beta_{4 k-1}<$ $x \leqq m+1$.

Now that we know the behavior of $E_{4 k-1}(x)$ on $[m, m+1]$ we are ready to prove the theorem. We know that $E_{4 k}(m)>0, E_{4 k}(m+1)>0$ and by (3.2) and (2.9) we have $E_{4 k}(m+1 / 2)<0$. This last inequality follows from the fact that if $k \geqq m^{2}$ then $(4 k) !>2(4 m)^{4 k}$, which can be proved in a straightforward elementary way. Also $E_{4 k}^{\prime}(x)<0$ for $m \leqq x<$ $\beta_{4 k-1}$ and $E_{4 k}^{\prime}(x)>0$ for $\beta_{4 k-1}<x \leqq m+1$. It follows that $E_{4 k}(x)$ has exactly two real roots $\beta_{4 k}^{(1)}, \beta_{4 k}^{(2)}$ such that $\beta_{4 k}^{(1)}<\beta_{4 k-1}<m+1 / 2<\beta_{4 k}^{(2)}$.

We now continue in the same way for $E_{4 k+1}(x)$. We have $E_{4 k+1}(m)>$ $0, E_{4 k+1}(m+1 / 2)>0$ and $E_{4 k+1}(m+1)<0$. Also $E_{4 k+1}^{\prime}(x)>0$ for $m \leqq x<$ $\beta_{4 k}^{(1)}$ and $\beta_{4 k}^{(2)}<x \leqq m+1$, while $E_{4 k+1}^{\prime}(x)<0$ for $\beta_{4 k}^{(1)}<x<\beta_{4 k}^{(2)}$. Thus $E_{4 k+1}(x)$ has exactly one real root $\beta_{4 k+1}$ in $[m, m+1]$ and $m+1 / 2<$ $\beta_{4 k+1}<\beta_{4 k}^{(2)}$. We know that $E_{4 k+2}(m)>0, \quad E_{4 k+2}(m+1 / 2)>0$, $E_{4 k+2}(m+1)>0, E_{4 k+2}^{\prime}(x)>0$ for $m \leqq x<\beta_{4 k+1}, E_{4 k+2}^{\prime}(x)<0$ for $\beta_{4 k+1}<$ $x \leqq m+1$. Thus $E_{4 k+2}(x)>0$ for $m \leqq x \leqq m+1$. We know that $E_{4 k+3}(m)<0, E_{4 k+3}(m+1 / 2)>0, E_{4 k+3}(m+1)>0, E_{4 k+3}^{\prime}(x)>0$ for $m \leqq$ $x \leqq m+1$. Thus $E_{4 k+3}(x)$ has exactly one real root $\beta_{4 k+3}$ in $[m, m+1]$ and $\beta_{4 k+3}<m+1 / 2$. We know $E_{4 k+4}(m)>0, \quad E_{4 k+4}(m+1 / 2)<0$, $E_{4 k+4}(m+1)>0, E_{4 k+4}^{\prime}(x)<0$ for $m \leqq x<\beta_{4 k+3}, E_{4 k+4}^{\prime}(x)>0$ for $\beta_{4 k+3}<$ 
$x \leqq m+1$. Hence $E_{4 k+4}(x)$ has exactly two real roots $\beta_{4 k+4}^{(1)}, \beta_{4 k+4}^{(2)}$ in $[m, m+1]$ and $\beta_{4 k+4}^{(1)}<\beta_{4 k+3}<m+1 / 2<\beta_{4 k+1}<\beta_{4 k}^{(2)}$.

Case 2. $m$ even. In this case we first prove the theorem for $E_{4 k+1}(x)$, treating $E_{4 k+1}(x)$ in exactly the same way we treated $E_{4 k-1}(x)$ when $m$ was odd. The rest of the proof is entirely analogous to the proof of Case 1. That is, we first examine $E_{4 k-1}(x)$ and $E_{4 k}(x)$ on the interval $[m-1, m]$ and then show $E_{4 k+1}(x)$ satisfies the theorem on $[m, m+1]$. Once we know the behavior of $E_{4 k+1}$ on $[m, m+1]$ we can easily determine the behavior of $E_{4 k+2}(x), E_{4 k+3}(x)$ and $E_{4 k+4}(x)$ on $[m, m+1]$.

It is known that $E_{n}(x)$ is a positive increasing function when $x$ is sufficiently large, i.e., $x>x_{0}$. The next theorem gives us an upper bound for $x_{0}$.

THEOREM 3.4. The polynomials $E_{4 k+s}(x), s=1,2,3,4$, are positive increasing functions on $[k+1, \infty)$.

Proof. We have seen that the theorem is true for $k=1,2,3$. Assume it is true for all $m<k$, and suppose $k$ is even. By (3.3) and (3.5) we see that $E_{4 k+s}(k+1)>0$ for $s=1,2,4$ and we are assuming $E_{4 k}(k+1)$ is a positive increasing function on $[k, \infty)$. Thus the only difficulty is to show that $E_{4 k+3}(k+1)>0$. We shall use (3.1), inequality (2.8) and the inequality

$$
2(k-1)^{4 k+3}<2 \sum_{r=0}^{k-1}(-1)^{r}(k-r)^{4 k+3} .
$$

Thus if we can show that

$$
(4 k+3) !<[3(k-1)]^{4 k+3}, \quad k \geqq 4,
$$

then it follows that $E_{4 k+3}(k+1)>0$. We prove (3.9) by first verifying the case $k=4$ from tables and then observing that

$$
(3 k-2+a)(k+6-a)<(3 k-3)^{2}
$$

for $a=0,1, \cdots, k+5$, with $k \geqq 5$. The proof for $k$ odd is very similar.

Theorem 3.4 can almost certainly be improved. In fact we conjecture that the polynomials $E_{8 k+s}(x), 1 \leqq s \leqq 8$, are positive, increasing functions on $[k+2, \infty)$.

Because of (2.4), we see that if $B_{n}(x)$ has no root in $(m, \infty)$ then $E_{n-1}(x)$ has no root in $(2 m, \infty)$. Inkeri [2] has shown that if $(M, M+1)$ is the largest interval in which $B_{n}(x)$ has real roots then $M \sim n / 2 e \pi$ as $n$ approaches $\infty$. 
4. Restrictions on the roots of $E_{n}(x)$. Inkeri [2] has shown that the only possible rational roots of $E_{n}(x)$ are 0,1 , and $1 / 2$. In this section we show that other types of real and complex numbers cannot be roots of $\operatorname{En}(x)$. We shall use the following lemma.

Lemma 4.1. Suppose $f(x)$ is a polynomial and

$$
f(a+b \sqrt{c})=\left(a_{1}+\cdots+a_{k}\right)+\sqrt{c}\left(b_{1}+\cdots+b_{k}\right)
$$

where each $a_{l}$ and $b_{\imath}$ is a rational number and $c$ is a square-free integer, $c>1$ or $c<0$. Suppose there is a prime number $p$ and positive integers $j$ and $m$ such that either

(a) $p^{m} a_{l} \neq \equiv 0(\bmod p)$ and $p^{m} a_{h} \equiv 0(\bmod p)$ for $h \neq j$

or

(b) $p^{m} b_{l} \neq \equiv 0(\bmod p)$ and $p^{m} b_{h} \equiv 0(\bmod p)$ for $h \neq j$. Then we can conclude that $f(a+b \sqrt{c}) \neq 0$.

THEOREM 4.1. If a is a nonzero rational number and $c$ is a nonzero integer, $c \neq 1$, then $E_{n}(a \sqrt{c}) \neq 0$.

Proof. Brillhart [1] has proved that $E_{n}(x)$ has no roots of the form $\alpha i$ where $\alpha$ is real, so we may assume $|c|>1$. By (2.3) we see that if $n$ is even the only nonzero term of $E_{n}(x)$ with an even exponent is $x^{n}$. Dividing $E_{n}(a \sqrt{c})$ into its rational and irrational parts, we see that the rational part is $a^{n} c^{n / 2} \neq 0$. If $n$ is odd, then $x^{n}$ is the only term of $E_{n}(x)$ with an odd exponent and in this case the irrational part of $E_{n}(a \sqrt{c}) \neq 0$.

THEOREM 4.2. If $a$ and $b$ are nonzero rational numbers and $c$ is an even square-free integer, then $E_{n}(a+b \sqrt{c}) \neq 0$.

Proof. First suppose $c>0$. If $n=2 k$ we use (2.11) to break $E_{2 k}(a+b \sqrt{c}) /(2 k)$ ! into its rational and irrational parts. Let $b^{2} c=$ $b_{1} / b_{2} 2^{q}, a=a_{1} / a_{2} 2^{z}$, g.c.d. $\left(b_{1}, b_{2}\right)=1=$ g.c.d. $\left(a_{1}, a_{2}\right)$ (a negative value of $q$ or $z$ indicates a power of 2 in the numerator). Note that $q$ must be odd. We now use Lemma 4.1 with $p=2$.

Case 1. $z<0, q<0$. From (2.11) we see that the maximum power of 2 occurs in the denominator of the irrational part of

$$
E_{2 k}(a+b \sqrt{c}) /(2 k) ! \text { when } \quad r=k-1, \quad s=2 k-1
$$

To see this, first replace $C_{s}$ in $(2.11)$ by $2^{s+1}\left(1-2^{s+1}\right) B_{s+1} /(s+1)$, keeping 
in mind that $2 B_{2 m} \equiv 1(\bmod 2)$ for $m>0$. Since $q$ and $z$ are both negative, we see that $\left(b^{2} c\right)^{k-r}$ and $a^{2 r+1-s}$ contribute the smallest possible power of 2 to the numerator when $r=k-1$ and $s=2 k-1$. Notice that in this case the power of 2 dividing the product $(s+1) !(2 k-2 r-1)$ ! $(2 r+1-s)$ ! in the denominator is maximum. This is the kind of reasoning we use in the remaining two cases and in Theorems 4.3 and 4.4.

Case 2. $z>0,2 z>q$. The maximum power of 2 occurs in the denominator of the rational part when $r=k, s=0$.

Case 3. $q>0, q>2 z$. The maximum power of 2 occurs in the denominator of the rational part when $r=0, s=0$.

When $n=2 k+1$ we use the irrational part of (2.12) and the proof is similar. If $c<0$ we divide $E_{n}(a+b \sqrt{c}) / n$ ! into its real and imaginary parts and proceed as before.

THEOREM 4.3. Suppose $c$ is an odd square-free integer, $c \neq 1$, and suppose $a$ and $b$ are rational numbers reduced to their lowest terms, $a=a_{1} / a_{2}, b=b_{1} / b_{2}$. If $E_{n}(a+b \sqrt{c})=0$ then $a_{2}=b_{2}$ and g.c.d. $\left(a_{2}, c\right)=$ $1=$ g.c.d. $\left(b_{2}, c\right)$.

Proof. We shall use the notation $p^{x} \| y$ to mean $p^{x}$ divides $y$ while $p^{x+1}$ does not divide $y$. First suppose $n=2 k$. Suppose $p$ is a prime number and $p^{z} \| a_{2}, z>0$. We want to show that $p^{z} \| b_{2}$ and g.c.d. $\left(a_{2}, c\right)=1$. Suppose $p^{q} \| b_{2}^{2} c^{-1}$. We shall show that $q=2 z$, so $p$ does not divide $c$.

Case 1. $2 z>q$. Using (2.11), we examine the rational (or real) part of $E_{2 k}(a+b \sqrt{c}) /(2 k)$ !, and we see that the maximum power of $p$ in the denominator occurs when $r=k, s=0$. Note that in this case if $p^{m} \|(2 k)$ ! then $p^{m} \|(s+1)$ ! $(2 k-2 r)$ ! $(2 r-s)$ !. If $p^{h} \| 2 k+1$, there are some terms having the property that if $p^{m} \|(2 k+1)$ ! then $p^{m} \|(s+1)$ ! $(2 k-2 r)$ ! $(2 r-s)$ ! For terms of this type the highest power of $p$ in the denominator occurs when $r=k, s=p^{h}-1$, but this power of $p$ is still less than the power occurring when $r=k, s=0$.

Case 2. $q>2 z$. The maximum power of $p$ occurs in the denominator of the rational (or real) part of $E_{2 k}(a+b \sqrt{c}) /(2 k)$ ! when $r=0, s=0$.

Thus, by Lemma 4.1 , if $p^{z} \| a_{2}, z>0$, we must have g.c.d. $\left(a_{2}, c\right)=$ 1. Also we have shown that $p^{z} \| b_{2}$. Now suppose $p^{q} \| b_{2} c^{-1} q>$ 0 . We want to show $p^{a} \| a_{2}$.

Case 1. $2 z>q$. The maximum power of $p$ in the denominator of 
the rational (or real) part of $E_{2 k}(a+b \sqrt{c}) /(2 k)$ ! occurs when $r=k$, $s=0$.

Case 2. $q>2 z$. The maximum power of $p$ in the denominator of the rational (or real) part of $E_{k}(a+b \sqrt{c}) /(2 k)$ ! occurs when $r=0$, $s=0$. Thus by Lemma 4.1 we must have $z=q$. If $n=2 k+1$ we examine the irrational (or complex) part of $E_{2 k+1}(a+b \sqrt{c}) /(2 k+1)$ ! and the proof is similar.

It is perhaps worth noting that $E_{3}(x)$ has the roots $(1 \pm \sqrt{3}) / 2$ and $E_{4}(x)$ and $E_{5}(x)$ both have the roots $(1 \pm \sqrt{5}) / 2$. Thus there are polynomials $E_{n}(x)$ having roots of the form $a+b \sqrt{c}, c$ odd.

THEOREM 4.4. If $a$ and $b$ are nonzero integers then $E_{n}(a+b i) \neq 0$.

Proof. Suppose $E_{2 k}(a+b i)=0$ and let $a=a_{1} 2^{z}, b=b_{1} 2^{q}, a_{1}$ and $b_{1}$ odd. Again we use Lemma 4.1.

Case 1. $q=0$. We can assume $z>0$ by (2.2). Examining the real part of $E_{2 k}(a+b i) /(2 k)$ !, we see that the highest power of 2 occurs in the denominator when $r=0, s=0$.

Case 2. $q>0$. Again, by (2.2), we can assume $z>0$. We look at the imaginary part of $E_{2 k}(a+b i) /(2 k)$ ! and the highest power of 2 occurs in the denominator when $r=k-1, s=2 k-1$. The proof for $E_{2 k+1}(a+b i)$ is similar.

Using the same method, we can prove the following theorem.

THEOREM 4.5. If $a$ and $b$ are rational numbers and $c$ and $d$ are square-free positive integers of different parity, then $E_{n}(a \sqrt{d}+b \sqrt{c} i) \neq 0$.

It should be pointed out that Theorems 4.1, 4.2, 4.4 and 4.5 also hold for the Bernoulli polynomials $B_{n}(x)$. The proofs are entirely analogous to the proofs in this paper.

Of course many questions remain unanswered. We have not been able to determine, for example, whether or not $a+b i$ can be a root of $E_{n}(x)$ if $a$ and $b$ are rational numbers. The writer also feels that Theorem 3.4 and the lower bound $m^{2}$ in Theorem 3.3 can both be improved. It would also be interesting to know how the roots of $E_{n}(x)$ are distributed in the last interval for which it has real roots.

5. Acknowledgement. I wish to thank the referee for his helpful suggestions. In particular, he has pointed out that the paper "Computation of tangent, Euler, and Bernoulli numbers" by D. E. Knuth and T. J. Buckholtz (Mathematics of Computation, vol. 21, Oct. 
1967, pp. 663-688) contains a more extensive listing of Euler and Bernoulli numbers than the reference cited in this paper.

\section{REFERENCES}

1. J. Brillhart, On the Euler and Bernoulli polynomials, J. Reine Angew. Math., 234 (1969), $45-64$.

2. K. Inkeri, The real roots of Bernoulli polynomials, Annales Universitatis Turkuensis, Series A, Nr. 37, Turku (1959), 3-20.

3. C. Jordan, Calculus of Finite Differences, Chelsea Publishing Co., New York, 1950.

4. N. Nörlund, Vorlesungen über Differenzenrechnung, Chelsea Publishing Co., New York, 1954.

5. S. Selby, editor in chief, CRC Standard Mathematical Tables, 17th Edition, The Chemical Rubber Co., Cleveland, 1969.

Received December 2, 1975 and in revised form March 30, 1976.

Wake Forest UNiversity 




\section{Pacific Journal of Mathematics}

Vol. 64, No. 1

May, 1976

Walter Allegretto, Nonoscillation theory of elliptic equations of order $2 n \ldots \ldots \quad 1$

Bruce Allem Anderson, Sequencings and starters.................. 17

Friedrich-Wilhelm Bauer, A shape theory with singular homology .......... 25

John Kelly Beem, Characterizing Finsler spaces which are

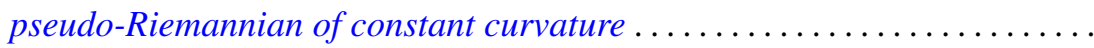

Dennis K. Burke and Ernest A. Michael, On certain point-countable

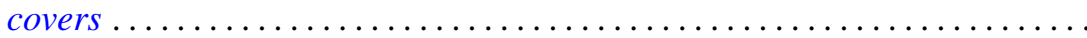

Robert Chen, A generalization of a theorem of Chacon ............... 93

Francis H. Clarke, On the inverse function theorem ................ 97

James Bryan Collier, The dual of a space with the Radon-Nikodým

property ....................................... 103

John E. Cruthirds, Infinite Galois theory for commutative rings ............ 107

Artatrana Dash, Joint essential spectra......................... 119

Robert M. DeVos, Subsequences and rearrangements of sequences in FK

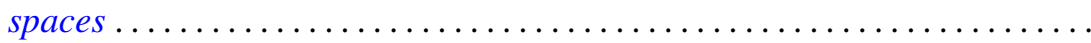

Geoffrey Fox and Pedro Morales, Non-Hausdorff multifunction generalization

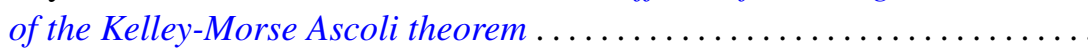

Richard Joseph Fleming, Jerome A. Goldstein and James E. Jamison, One

parameter groups of isometries on certain Banach spaces.............

Robert David Gulliver, II, Finiteness of the ramified set for branched

immersions of surfaces

Kenneth Hardy and István Juhász, Normality and the weak cb property ...... 167

C. A. Hayes, Derivation of the integrals of $L^{(q)}$-functions.

Frederic Timothy Howard, Roots of the Euler polynomials .

Robert Edward Jamison, II, Richard O'Brien and Peter Drummond Taylor, On

embedding a compact convex set into a locally convex topological vector space ....................................

Andrew Lelek, An example of a simple triod with surjective span smaller than span ...

Janet E. Mills, Certain congruences on orthodox semigroups

Donald J. Newman and A. R. Reddy, Rational approximation of $e^{-x}$ on the positive real axis.

John Robert Quine, Jr., Homotopies and intersection sequences ...

Nambury Sitarama Raju, Periodic Jacobi-Perron algorithms and fundamental units ....

Herbert Silverman, Convexity theorems for subclasses of univalent functions. . .

Charles Frederick Wells, Centralizers of transitive semigroup actions and endomorphisms of trees.........................

Volker Wrobel, Spectral approximation theorems in locally convex spaces ..................... 